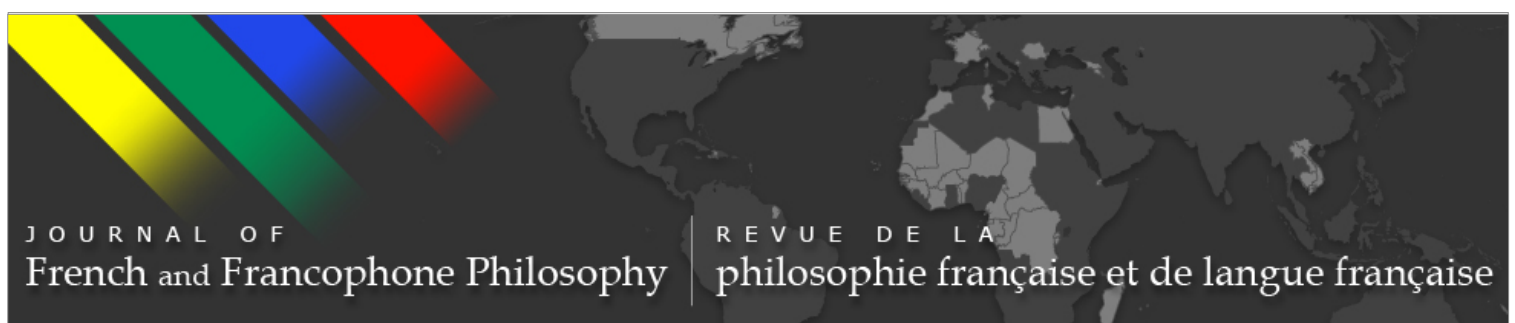

\title{
Claude Romano au carrefour de la phénoménologie française
}

\section{Carla Canullo}

Journal of French and Francophone Philosophy - Revue de la philosophie française et de langue française, Vol XXI, No 2 (2013) pp 87-104

\author{
Vol XXI, No 2 (2013) \\ ISSN 1936-6280 (print) \\ ISSN 2155-1162 (online) \\ DOI $10.5195 /$ jffp. 2013.605 \\ www.jffp.org
}

\section{(oc) BY-NG-ND}

This work is licensed under a Creative Commons Attribution-Noncommercial-No Derivative Works 3.0 United States License.

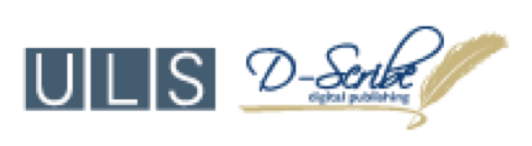

This journal is operated by the University Library System of the University of Pittsburgh as part of its D-Scribe Digital Publishing Program, and is co-sponsored by the University of Pittsburgh Press 


\title{
Claude Romano au carrefour de la phénoménologie française
}

\author{
Carla Canullo
}

Université de Macerata

Depuis la fin des années '90, la réflexion sur l'événement a permis de compter Claude Romano parmi les protagonistes de la phénoménologie française contemporaine. Sa proposition phénoménologique s'est ensuite nouée (grâce à l'endurante lecture des romans de Faulkner) à l'inouï débordement de l'événement de la vie que le récit est censé redonner, pour ainsi dire, "en elle-même" et à l'abri de toute sorte de réduction. ${ }^{1}$

L'enjeu de ces pages - consacrées à l'ouvrage paru en 2010, Au cœur de la raison, la phénoménologie ${ }^{2}$ - est la même répétition de la phénoménologie que Romano nous a livrée dans cet ouvrage. En effet, notre avis est que Romano, en 2010, a pratiqué une Wiederholung de la phénoménologie et qu'il l'a fait en situant celle-ci au centre d'un carrefour où confluent les questions historiques (Husserl, Heidegger), les libres variations de la phénoménologie en France (Sartre, Merleau-Ponty, Levinas) et la philosophie analytique. Or, par cette répétition, il s'est installé au carrefour de la phénoménologie française contemporaine, et cela pour deux raisons: premièrement, parce qu'il a donné une contribution décisive à l'alternative "réduction vs intentionnalité" qui ne cesse de représenter l'alternative de la réception française de la phénoménologie. ${ }^{3}$ Deuxièmement, parce que par sa répétition il a ouvert aussi un chemin nouveau et original qui maintenant demande à être phénoménologiquement questionné.

\section{Première confluence: l'épochè entre cartésianisme et anti-cartésianisme}

"D'autant plus de réduction, d'autant moins de donation": c'est le commentaire lapidaire de Marc Richir au $\S 8$ du manuscrit de Husserl n. $31 .{ }^{4}$ L'opposition vis-à-vis du principe de Jean-Luc Marion "autant de réduction, autant de donation" ${ }^{5}$ est patente. Pourtant, ce n'est pas l'opposition entre les deux démarches qui nous intéresse car notre intérêt porte sur la voie phénoménologique que l'on fraye lorsqu'on ne privilégie pas le lien justement célèbre - entre Reduktion et Gegebenheit, mais lorsqu'on radicalise

Journal of French and Francophone Philosophy | Revue de la philosophie française et de langue française Vol XXI, No 2 (2013) | www.jffp.org | DOI 10.5195/jffp.2013.605 
88 Claude Romano au carrefour de la phénoménologie française

l'épochè jusqu'à la réduction de toute intentionnalité possible. ${ }^{6}$ A ce propos Richir écrit:

Alors que l'épochè phénoménologique classique (ou "standard") de Husserl consiste à suspendre et à surprendre, comme ils se donnent avec les objets, les différents types de doxa pour en analyser les structures, l'épochè phénoménologique ("non-standard") , c'est-à-dire l'épochè phénoménologique hyperbolique, consiste à suspendre et à surprendre, à même les apparitions et les apparaissants, les structures intentionnelles elles-mêmes [...] pour accéder à ce que nous nommons le phénomène comme rien que phénomène. ${ }^{7}$

De façon très efficace, dans son commentaire de Husserl que nous avons cité Richir écrit:

Le phénomène se phénoménalise en clignotant, en battant en éclipses, comme le constituant dans le constitué, entre son apparition qui, si elle allait à son terme, ferait disparaître le constituant dans un constitué quelconque, et sa disparition qui, si elle allait à son terme, ferait disparaître le constituant dans ce constitué déterminé dont le constituant paraît constituant - cela montre en quoi le clignotement phénoménologique ne peut s'arrêter que dans l'abstraction corrélative de l'illusion transcendantale phénoménologique, les deux pôles du clignotements demeurant écartés comme l'indéterminé et le déterminé. 8

Enfin, le phénomène rien que phénomène ne se montre que par la distance infinie du phénomène en soi-même, ${ }^{9}$ ce qui nous reconduit aux alentours de la radicalité d'une épochè phénoménologique hyperbolique qui perce vers "le clignotement phénoménologique en quoi consiste [...] la réduction phénoménologique: réduction au phénomène comme rien que phénomène qui clignote entre son apparition et sa disparition."10

Le passage que l'on vient de citer explique - au moins c'est notre avis le sens du principe "d'autant plus de réduction, d'autant moins de donation" car ce clignotement nous semble provenir moins de la Gegebenheit husserlienne que de la Ungegebenheit d'Eugen Fink. En effet, celui-ci, vis-àvis de la "phénoménologie régressive" que Husserl expose dans les Méditations cartésiennes 1-5, dans la VI Méditation cartésienne présente une "phénoménologie constructive" d'après laquelle "la donation du thème à l'activité phénoménologisante est une non-donation (Ungegebenheit), l'être là présent du moi théorisant est à proprement parler un ne pas être là présent." ${ }^{11}$ Et peu loin: "L'être qui peut être dégagé sous la forme d'une construction ne possède pas par principe un "spectateur" qu'il a établi lui-même; la vie

Journal of French and Francophone Philosophy | Revue de la philosophie française et de langue française

Vol XXI, No 2 (2013) | http://www.jffp.org | DOI 10.5195/jffp.2013.605 
transcendantale non-donnée ne peut "venir à elle-même" que dans le spectateur de la vie transcendantale donnée."12

Le moi phénoménologisant, transzendentale Zuschauer, naît par l'acte de l'épochè qui produit une fracture dans la vie transcendantale avec laquelle le moi phénoménologisant demeure en contact tout en restant en même temps en stricte contact avec le monde. En effet, "lorsque nous phénoménologisons, nous sommes toujours déjà rapportés à une constitution du monde que se trouve être en cours, mais nous ne sommes absolument jamais rapportés intuitivement à une constitution du monde qui vient tout au juste d'être mise en jeu ou qui vient à peine de s'arrêter." ${ }^{13}$ Ne sommes-nous pas face au jeu paradoxal de Gegebenheit et Ungegebenheit? Oui, tout comme Fink l'explique, en soulignant aussi que "la vie transcendantale non-donnée ne peut "venir à elle-même" que dans le spectateur de la vie transcendantale donnée." ${ }^{14}$ Dès lors, en-deçà de toute opposition polémique, la descendance finkienne du principe "d'autant plus de réduction, d'autant moins de donation" est évidente. Cela dit, revenons au texte de Husserl Intentionnalité et être-aumonde et au commentaire de Richir, parce que, dans le cadre de cette radicalisation de l'époquè en amont, l'intentionnalité fait à nouveau mais en aval son apparition.

Dans ce texte, Husserl aborde la question du "dedans" et du "dehors" caractérisant en propre l'intentionnalité, une question sur laquelle même Romano revient à maintes reprises dans son ouvrage. En abordant ce sujet, le fondateur de la phénoménologie emploie au moins deux fois le mot Rätsel; d'abord lorsqu'il parle de la séparation entre "représentation du monde" et "monde," ${ }^{15}$ deuxièmement lorsqu'il pose la question: "Comment l'homme connaissant dans son immanence de connaissance peut-il se transcender luimême, devenir même conscient d'un être transcendantal?"16 Il s'agit de l'énigme de l'objectivité de la connaissance, une énigme insoluble qui nous oblige à ne pas oublier que "mon et notre être humain [...], avec tout connaître et tout connu, devient à présent universellement problématique en tant que mondain." ${ }^{17}$ Et plus loin, on lit que "c'est dans l'essence générale de l'intentionnalité transcendantale que repose déjà cela que le dedans et le dehors ne s'excluent pas, mais s'exigent. La vie intentionnelle dans son écoulement est une effectuation (Leisten) constante à travers la modification intentionnelle de modes multiples. Toute modification intentionnelle constitue un dehors dans le dedans." ${ }^{18}$

Or Richir discerne dans ce "dehors dans le dedans" le rien que phénomène et il vise dans l'intentionnalité husserlienne la découverte concernant que "ce dehors transcendantal est le dedans phénoménologique $d u$ "dedans" (apparemment psychique), ou que ce dedans du dedans, comme interfacticité transcendantale constituante, est un dehors par rapport à tout donné." ${ }^{19}$ La conclusion que Richir tire de ces passages est que le "dehors" phénoménologique n'est rien d'autre que le phénomène comme rien que phénomène. Une sorte d'entrelacs osmotique entre dedans et dehors clignote ici - dirait-on en empruntant les mots de 
90 Claude Romano au carrefour de la phénoménologie

française

Richir; en outre, c'est dans ce passage que l'on comprend le sens d'une épochè hyperbolique, censée réduire aussi bien le psychique que l'extrapsychique à la dimension transcendantale, ce qui n'empêche pas que se pose la question concernant le "comment" la différence entre ces deux dimensions (dehors-dedans) soit gardée.

Or Romano cite maintes fois le nom de Fink et parmi ces citations nous concentrons notre attention sur celles où il aborde le sujet qui nous occupe, l'épochè. L'avis de notre auteur est pourtant que celle-ci, chez Fink, n'a rien d'hyperbolique et que loin de suspendre tout jugement vis-à-vis du monde elle ménage notre accès à celui-ci. ${ }^{20}$ En plus, l'épochè n'est aucunement suspension du jugement, mais elle concerne une "croyance spontanée et préthéorique à l'être au monde"; ou encore, elle est pleinement universelle et, "comme Fink l'a remarqué, est-elle tout proche de l'étonnement, car elle nous met aux prises avec l'énigme du monde (Welträtsel). Enigme d'une transcendance dont il s'agit de prendre la mesure, et non point problème celui de l'existence du monde - qu'il s'agirait de résoudre." ${ }^{21}$ C'est encore en citant Fink ${ }^{22}$ que, un peu plus loin, Romano revient à nouveau sur cette capacité de l'épochè de ménager notre accès au monde:

Comme l'indique Eugen Fink [...] l'épochè n'est pas une délimitation (Einschränkung) de la conscience, mais une délimitation (Entschränkung) de celle-ci, c'est-à-dire une illimitation au terme de laquelle les concepts mêmes d'intérieur et d'extérieure deviennent problématiques. La perte du monde, sa mise hors-jeu en tant que sol de l'attitude naturelle, n'est alors rien d'autre que la découverte $\mathrm{du}$ monde, [...] la réduction, c'est le monde perdu et retrouvé. ${ }^{23}$

La proximité avec le dedans et le dehors husserliens commentés par Richir est patente. Cependant, la stratégie d'interprétation est tout à fait différente car la question phénoménologique qui occupe Romano n'est pas celle de l'ancrage transcendantal de ces deux dimensions mais, au contraire, il questionne la réunion possible entre monde et conscience parce que cette même réunion est et ne peut être que structurelle: elle est "son intentionnalité," ${ }^{24}$ à savoir l'intentionnalité de la conscience. Et pourtant, le cœur de la lecture de Romano n'a pas été atteint si on méconnaît que son but est la critique du cartésianisme conçu comme la seule interprétation véritable de la phénoménologie husserlienne et, en générale, de la phénoménologie. ${ }^{25}$ Or ce cartésianisme qui est sans doute un trait de la phénoménologie husserlienne, représente aussi le destin de la phénoménologie en tant que telle.

C'est par un long détour à travers les textes husserliens que Romano formule sa réponse négative, aussi bien vis-à-vis de cette interprétation cartésienne de la phénoménologie que de la figure de raison qu'elle ébauche. "La tentation cartésienne triomphe au $\S 49$ des Ideen... I, dans lequel la

Journal of French and Francophone Philosophy | Revue de la philosophie française et de langue française Vol XXI, No 2 (2013) | http://www.jffp.org | DOI 10.5195/jffp.2013.605 
disparité ontologique de la conscience et la réalité est révélée à travers l'hypothèse de l'anéantissement du monde [...]; même si un tel cataclysme se produisait, affirme Husserl, il n'affecterait en rien l'existence de la conscience." ${ }^{26}$ Partageant avec Rudolf Bernet l'idée que chez Husserl il y aurait un "dualisme ontologique dont les termes seraient d'un côté l'être évident de la conscience et, de l'autre côté, l'être dubitable du monde,"27 Romano propose de renverser la thèse selon laquelle "pour comprendre le motif transcendantal (husserlien, ndlr.) en sa pureté, il faudrait abandonner ce dualisme," 28 en tirant la conclusion que la compréhension correcte de la thématique transcendantale présuppose le dualisme et non le contraire. En effet, chez Husserl, il y aurait une tension entre conscience et monde (autre que la conscience) et même l'épochè supposerait ce dualisme, car "la suspension de la thèse de l'attitude naturelle, ne pourrait reconduire à aucun ego transcendantal constituant [...] si l'ego n'était conçu comme une instance antérieure en droit au monde au double sens d'une antériorité ontologique et épistémique, donc si l'ego ne possédait une autarcie existentielle qui le rend indépendant de l'existence du monde." ${ }^{29}$ Or c'est dans la ligne husserlienne/cartésienne que, sous certains aspects, Richir se pose lorsqu'il nomme hyperbolique son épochè et qu'il évoque le Malin Génie de Descartes. ${ }^{30}$ De plus, selon Richir, cette épochè radicale est la condition de possibilité du clignotement phénoménologique, voire de l'apparition du phénomène rien que phénomène. ${ }^{31}$

Nous voici donc au carrefour de la phénoménologie française, plus précisément au croisement de deux interprétations de la phénoménologie, l'une radicalisant l'épochè - plus cartésienne que le doute cartésien (Richir) l'autre anticartésienne (Romano). L'une qui provient de l'interprétation transcendantale de la phénoménologie (Richir) et qui aboutit au dépassement de l'intentionnalité, l'autre qui s'oppose à cette même interprétation et à la préséance du cogito (et donc du motif cartésien de la phénoménologie), en provenant des réflexions consacrées à l'advenant, c'est-àdire à celui qui, en disqualifiant toute pensée du sujet, n'est "celui à qui quelque chose est arrivé (le "sujet" d'assignation des événements) que pour autant et dans la mesure où il est d'abord et plus originairement celui à qui échoient des événements, capable de se comprendre lui-même à partir d'eux, c'est-à-dire, rigoureusement, de s'advenir lui-même à partir d'eux." ${ }^{32}$ A la stricte rationalité cartésienne fait donc écho ce premier élargissement du "sujet de la raison" que la phénoménologie herméneutique événementielle de Romano présentait déjà en 1998. A cette époque, l'élargissement était radical parce qu'il questionnait la primauté égologique en accomplissant un pas, pour ainsi dire, vers le fond, c'est-à-dire vers la racine événementielle du sujet/advenant à l'encontre du pas en avant heideggérien - voire à l'encontre de l'ouverture des possibles du Dasein résolu. ${ }^{33}$ Cependant, une fois expliqué d'où provient le motif de la critique anticartésienne de Romano, il faut maintenant discerner vers où perce sa proposition phénoménologique. 
92 Claude Romano au carrefour de la phénoménologie

\section{L’intentionnalité en question}

L'intentionnalité en question est le titre du volume collectif édité par Dominique Janicaud en $1995 .{ }^{34}$ Romano y pourrait aujourd'hui ajouter un autre chapitre, une sorte d'Appendice ou de prolongement des textes recueillis. En effet, c'est vers une relecture de l'intentionnalité que perce son ouvrage, tout comme le montrent les quatre chapitres consacrés au "thème capital de la phénoménologie" ${ }^{35}$ où la lecture critique de Husserl s'enchevêtre avec la proposition phénoménologique de Romano. Entre l'intentionnalité husserlienne qui reste toujours dans l'entre-deux du même dualisme cartésien signalé à propos de l'épochè ${ }^{36}$ et la proposition merleaupontienne de l'intentionnalité de la perception ainsi que de son primat, Romano choisit la seconde démarche. ${ }^{37}$ Il ne s'agit pas d'une préférence de style (Merleau-Ponty vs Husserl), mais d'un véritable passage phénoménologique qui découle strictement de ce que l'on a dit plus haut à propos de l'épochè. Encore, il s'agit d'un passage phénoménologique qui, au lieu de privilégier un seul motif phénoménologique (épochè/réduction vs intentionnalité), vise à saisir l'enchevêtrement des deux thèmes pour atteindre le cœur du déploiement phénoménologique lui-même, ou bien du mouvement de la phénoménologie en tant que telle. Il s'agit du mouvement anticopernicien sur lequel se conclut l'ouvrage de 2010, un mouvement qui pourtant résulte d'un geste interprétatif préalable concernant la conception husserlienne du monde:

Comme par un tour de passe-passe, la réduction n'a pas seulement changé notre point de vue sur le monde; elle a modifié ce que nous entendions par "monde" antérieurement à son accomplissement. Appelons "monde prédonné" le monde de l'attitude naturelle [...]. Appelons "monde constitué" le monde tel qu'il nous apparaît après la réduction. Le premier est indépendant de notre connaissance; le second n'a aucun être en dehors du processus de sa constitution, donc de sa connaissance dans et par l'ego. Ces deux mondes, nous dit Husserl en substance, n'en font qu'un seul: le monde prédonné est le monde constitué. Mais comment cela est-il possible, si le premier se définit par son indépendance et le second par sa dépendance vis-à-vis de la conscience? Husserl a moins résolu le problème de l'intentionnalité, il a moins montré positivement comment la conscience peut sortir d'elle-même pour atteindre la réalité, qu'il n'a rendu la réalité entièrement dépendante de la conscience au point d'en devenir le produit intentionnel. ${ }^{38}$

Dans son interprétation des textes husserliens, Romano remarque que le cœur de l'intentionnalité est dans le fait que celle-ci est relation ${ }^{39}$ et, cela dit,

Journal of French and Francophone Philosophy | Revue de la philosophie française et de langue française

Vol XXI, No 2 (2013) | http://www.jffp.org | DOI 10.5195/jffp.2013.605 
il n'est pas difficile de comprendre le sens de cette critique à Husserl. Si le souci transcendantal de la phénoménologie husserlienne "réduit" le "monde prédonné" au "monde constitué," seule la relation entre ces "deux mondes" gardée à l'abri de toute épochè et réduction transcendantales - nous donne l'assurance que cet accès est possible. En plus, seule une intentionnalité qui ne répète pas la même épochè/réduction de la compréhension transcendantale et qui demeure "autre" vis-à-vis de celle-ci, sans pour autant aboutir à un irrationalisme sauvage, peut ménager notre accès au monde. Et pourtant, cette relecture de l'intentionnalité - qui "place" à nouveau Romano au carrefour de la phénoménologie française - est-elle le résultat d'un croisement syncrétique de positions ou bien est-elle un lieu de convergence, voire un seuil où on ne peut que passer pour se diriger ailleurs?

Certes, un carrefour risque vi propria sua la convergence syncrétique des positions. Pourtant, l'auteur ne nous lègue aucunement une lecture synoptique ni syncrétique, car son analyse phénoménologique se développe en répondant aux questions que la "phénoménologie historique" pose, mais aussi que la philosophie analytique conteste et que Romano aborde "en première personne." Cependant le risque demeure là, tout comme l'atteste le fait que le syncrétisme ne concerne pas la seule relecture exposée à propos de l'épochè/réduction mais encore cet autre grande question, l'intentionnalité. En effet, le propos de "libérer l'intentionnalité de la compréhension transcendantale" est aussi le but de la voie phénoménologique qu'un autre protagoniste de la phénoménologie française, Renaud Barbaras, a parcouru dans le sillage de Merleau-Ponty et Patočka. En outre, Le chant de la vie. Phénoménologie de Faulkner ${ }^{40}$ aurait pu faire écho aussi bien à Vie et intentionnalité. Recherches phénoménologiques ${ }^{41} \mathrm{de}$ Barbaras qu'à la phénoménologie de la vie de Michel Henry. Le reproche de syncrétisme guette donc toujours et la ligne de démarcation entre une véritable Wiederholung et une lecture synoptique/syncrétique est très subtile, d'autant plus que la première page de Vie et intentionnalité de Barbaras pose vis-à-vis de l'intentionnalité husserlienne presque les mêmes questions que pose Romano. En outre, la voie empruntée aussi bien par notre auteur que par Barbaras les conduit à l'écoute de Merleau-Ponty. En sorte que cette phrase pourrait s'inscrire aussi bien dans un ouvrage de Romano que de Barbaras:

$C^{\prime}$ est donc bien à la condition de se défaire du modèle de l'objet, c'est-à-dire de penser la perception à partir d'ellemême, que l'on se trouve en mesure de rendre compte de l'intentionnalité: si la perception se rapporte à autre chose qu'elle-même, c'est justement parce que ce qu'elle vise n'est pas un objet mais une transcendance ou une profondeur pure. $^{42}$

De même, l'intentionnalité n'est pas le seul motif où ces voies du carrefour se croisent. Barbaras cherche dans la phénoménologie de Merleau-Ponty et

Journal of French and Francophone Philosophy | Revue de la philosophie française et de langue française 
94 Claude Romano au carrefour de la phénoménologie

française

de Patočka la réponse à la séparation husserlienne entre sens et existence incarnée et c'est à ce propos que l'intentionnalité joue un rôle fondamental, car c'est à elle que revient la possibilité de nous faire accéder au monde incarné à l'encontre de toute dés-incarnation du transcendantal. Patočka est le seul qui, selon Barbaras, a cherché à intégrer existence et corporéité sans "référer cette corporéité à la présence-subsistante" ${ }^{43}$ - et cela à l'écart de Merleau-Ponty. En outre, Patočka sortirait de l'alternative "ou bien la chair, ou bien le sens" grâce au mouvement de la vie et à la "détermination de l'intentionnalité perceptive comme mouvement, l'identité pure et simple du se-mouvoir et du percevoir." 44 Et plus loin, en poursuivant dans son commentaire croisé de Merleau-Ponty et Patočka, il écrit:

En raison de sa distance ou de sa profondeur constitutive, le pôle de l'intentionnalité perceptive doit [...] être caractérisé comme monde, comme cet Être vertical que toutes les perceptions atteignent et qu'aucune n'épuise. La question est donc bien celle de l'intentionnalité (ou de l'existence) en tant qu'elle est capable de se rapporter à un monde, c'est-à-dire finalement à ce qui porte tout étant sans relever soi-même de l'étant, à ce qui est plus qu'objet [...] et ne peut, pour cette raison même, être réintégré à la sphère du sujet. [...] Il faut donc comprendre l'intentionnalité comme une activité qui est fondamentalement passivité puisqu'elle n'atteint le monde que comme cela qu'elle ne peut justement posséder comme une puissance impuissante ou comme la puissance propre de l'impuissant. ${ }^{45}$

Patočka nous apprend que le mouvement de la vie est le "véritable tissu de l'intentionnalité perceptive" 46 et qu'il est même "l'irréductible coappartenance de l'existence et de la vie." ${ }^{47}$ Or cela n'est possible que parce que l'intentionnalité se caractérise par le fait d'être traversée "par la déhiscence de l'existence et de la vie," ce qui rend possible "de référer le mouvement à la source plus profonde qui rend compte de ce redoublement interne par lequel il n'est pas dépassement vers le monde qu'en se dépassant en tant que mouvement purement vivant." ${ }^{48}$ Enfin, à la question "comment définir ultimement l'intentionnalité en tant qu'en elle la vie est aussi existence?," 49 Barbaras répond que l'essence du mouvement vivant est le Désir qui, en même temps, est manque et aspiration, défaut et satisfaction. ${ }^{50}$ Dès lors, c'est bien par ce Désir, "autre nom de l'intentionnalité" que "le sujet en vient à se constituer comme le corrélat de ce rien qui l'anime, c'est-à-dire comme étant en question pour lui-même." ${ }^{51}$

Or l'intentionnalité dont Romano parle perce ailleurs pour répondre, pourtant, aux mêmes questions. Nous disons qu'elle perce ailleurs parce

Journal of French and Francophone Philosophy | Revue de la philosophie française et de langue française

Vol XXI, No 2 (2013) | http://www.jffp.org | DOI 10.5195/jffp.2013.605 
qu'une fois que son sens a été atteint, on déclare son insuffisance, son défaut et son manque, sans pourtant que ceux-ci soient conçus selon le même sens que celui que Barbaras propose: il s'agit plutôt d'une insuffisance à exprimer ce que nous discernons comme les irréductibles de la phénoménologie de Romano, à savoir l'ouverture au monde et l'expérience. Notre avis est que ces irréductibles représentent ce que Romano envisage par sa quête du double holisme de l'horizon et du monde. ${ }^{52}$ En effet si "le modèle de l'intentionnalité suppose [...] que l'on puisse isoler un contenu intentionnel qui appartienne à un acte intentionnel donné," ${ }^{53}$ comment expliquer l'unité qui fait qu'une perception soit une perception dans un ensemble? L'intentionnalité - supposant vi propria sua une relation - isole pourtant les éléments de cette relation qui n'en envisagent pas la totalité capable d'en rendre compte. Dès lors, au carrefour de la phénoménologie française, Romano emprunte une voie à lui, originale et qui, après l'avoir conduit au-delà du cartésianisme, le conduit au-delà du thème capital de la phénoménologie, car l'intentionnalité - même l'intentionnalité perceptive - échoue et n'arrive pas jusqu'à l'ouverture du monde. Cela arrive, pourtant, pour des raisons radicalement opposées vis-à-vis de celles aperçues à propos de l'épochè, car si celle-ci n'était pas capable de ménager notre accès au monde par "défaut de monde," l'intentionnalité échouerait par "manque du tout holistique du monde." Ainsi, l'une échoue parce qu'elle aboutit à la réduction transcendantale du "dehors-dedans" en creusant un abîme entre le monde et le transcendantal, l'autre parce qu'elle aboutit à la méconnaissance du tout holistique, ou bien du "caractère radicalement holistique de notre être-aumonde." 54

Or c'est pour expliquer cet holisme de l'être-au-monde que Romano radicalise l'intentionnalité en la concevant "comme signification inhérente à la chose et offerte à une intelligence pratique que l'on peut appeler "compréhension" à condition de tenir ce terme au plus loin de l'idée d'une appréhension intellectuelle et discursive." ${ }^{55}$ Quoi de neuf, pourtant dans ce questionnement? Lorsque Barbaras remarque que l'intentionnalité est un sens d'être spécifique et que Heidegger "peut voir dans l'être-au-monde [...] la signification véritable de l'intentionnalité," ${ }^{56}$ ne posait-il pas les mêmes questions que Romano? Et le carrefour, ne risque-t-il pas de se transformer en un cercle vicieux découlant du fait que l'on ne pose que les mêmes questions? On ne sort pas de cet impasse si on méconnaît que le carrefour est, certes, un lieu de croisement mais aussi un non-lieu où "on passe" afin d'emprunter une autre voie. Or la voie que Romano emprunte lui permet d'atteindre de façon radicalement anticartésienne une question radicalement phénoménologique: la possibilité qu'un irréductible se donne.

\section{L'irréductible en deçà de la réduction}

Lors d'une première rencontre avec Romano et sa pensée, organisée en 2005 à Paris à l'Institut Catholique, nous posions une question à l'ensemble des ouvrages consacrés à l'événement: si celui-ci est une des modalités pour

Journal of French and Francophone Philosophy | Revue de la philosophie française et de langue française 
penser l'irréductible, ne faut-il pas introduire, afin que l'on parle bien de l'irréductible, une figure de réduction, ce que pourtant Romano foncièrement conteste?

Rappelons brièvement ce que nous entendions (et entendons) par irréductible. Celui-ci se caractérise par le fait d'être irrenonçable, en s'écartant d'une "optionalité" philosophique qui finirait par empêtrer la pensée dans des alternatives de principe. ${ }^{57}$ Même si on ne peut pas ignorer qu'à l'origine de n'importe quel geste philosophique il y a un choix, une "option pour," une voie plutôt qu'une autre, on doit reconnaître que s'interroger sur un irréductible revient à chercher le motifépistémologique qui est le non-fond-en tant que condition de possibilité - du surgissement même de l'option. Nous appelons ce motif "épistémologique" en indiquant par cet adjectif le fait qu'il est une condition de possibilité que "quelque chose" se donne à la pensée. Or si l'irréductible est cette condition de possibilité, il doit être aussi irrenonçable, car si on y renonce, on renonce aussi à ce qui rend possible le fait même de penser. Mais irrenonçable ne veut toutefois pas dire irrefusable, parce que l'on peut bien se refuser à l'irréductible.

Cela dit, revenons à notre question: comment est-il possible de parler d'irréductible sans pratiquer la réduction? Certes, il ne faut pas ignorer qu'en 2010 Romano pose d'autres questions que celles qu'il posait à propos de l'événement et que, dans sa proposition phénoménologique, un rôle massif revient à la lecture de la philosophie analytique. Pourtant, a-t-il atteint aussi le cœur phénoménologiquement irréductible - donc irrenonçable mais non irrefusable - de la raison? Notre avis est que oui, et nous pensons qu'il l'a atteint et que cela représente aussi la voie originale qu'il a emprunté et qui le fait sortir du carrefour de la phénoménologie française. Ce qui sera d'ailleurs expliqué par le recours à ce qui ouvre et conclut l'ouvrage du 2010, l'expérience. Loin de constituer une petitio principiis ou une boucle étrange telle que celle que Hofstadter a dévoilée, ${ }^{58}$ notre avis est que partir de l'expérience pour y revenir décèle l'irrenonçable irréductibilité de l'expérience-même car rien ne se donne si celle-ci n'advient pas. Toutefois, les volumes sur l'événement ne proposaient rien de différent: ${ }^{59}$ à quoi bon ce long détour phénoménologique pour répéter ce que l'on connaissait déjà?

Or notre avis est que loin d'être une petitio principiis ou une boucle étrange, l'expérience est plutôt la forme de la Wierderholung phénoménologique de Romano. Autrement dit, au carrefour de la phénoménologie notre auteur ne répète pas la phénoménologie en tant que telle $^{60}$ ni ne propose des "nouvelles fondations" ${ }^{161}$; il offre $-c^{\prime}$ est au moins notre libre interprétation - une traversée de ce que fait l'homme tel qu'il est car il n'y a de raison qui ne soit aussi et en même temps "raison d'un homme," ou bien de l'homme. En effet, c'est bien le sens de cette Wierderholung phénoménologique que l'on comprend si on conjoint l'ouvrage de 1998 avec celui de 2010, c'est-à-dire cet arc qui va de l'expérience événementielle à l'expérience au cœur de la raison.

Journal of French and Francophone Philosophy | Revue de la philosophie française et de langue française Vol XXI, No 2 (2013) | http://www.jffp.org | DOI 10.5195/jffp.2013.605 
"L'essence" de l'homme est l'ex-pér-ience, c'est-à-dire la capacité de s'advenir singulièrement à travers ce qui lui arrive, et d'apparaître par conséquent irréductible à toute essence générique. [...] L'ex-pér-ience en nous singularisant, nous ouvre à la singularité de chaque humain dans ce qu'elle a d'irréductible. [...] Si l'universalité de l'humain n'est accessible qu'à travers l'expér-ience singulière, c'est parce que l'humanité même (l'universel), c'est l'ex-pér-ience, c'est-à-dire la capacité de devenir soi-même (singulier) à travers l'épreuve de ce qui arrive - de sorte que, si l'humain nous est accessible, c'est non seulement à partir de l'ex-pér-ience, mais comme cette ex-pér-ience même, et pour autant qu'elle nous singularise. C'est en devenant moi-même que je comprends l'humanité, en moi et hors de moi, non pas au sens d'une connaissance théorétique, extérieure pour ainsi dire à son objet, mais au sens d'une compréhension qui n'est donnée que par l'épreuve, en tant que traversée et endurance de l'humain: ex-pér-ience au sens premier et originaire. $^{62}$

Plus loin, Romano écrit ainsi que

l'humanité de l'homme est ex-pér-ience, capacité de se singulariser, formellement synonyme de l'ipséité en son sens événemential. Être homme, c'est être capable de devenir soi, et être soi, c'est être capable de devenir homme, s'approprier son propre en le devenant, d'une manière irréductiblement unique. Ce qui revient à dire aussi qu'être homme, c'est pouvoir être soi, avoir une ipséité. $^{63}$

Le cœur de ces passages est, certes, l'événement, et notamment celui de la naissance. ${ }^{64}$ Pourtant un lien se dessine entre ces pages et celles que Romano écrit dix ans après, car "expérience" et "capacité" sont aussi les motifs phénoménologiques de l'ouvrage du 2010. En critiquant le choix heideggérien de "résorber la diversité des capacités dans (la) seule capacitéà-être et, ce faisant, de résorber le monde naturel dans le monde existential"65 et en essayant de "retrouver" le monde naturel, Romano met en œuvre une répétition des capacités de l'homme à l'aune aussi bien de l'inscription de celui-ci dans le naturel que de son exposition à l'événement. ${ }^{66}$ Et cela manifeste une sorte de hiérarchisation de ces mêmes capacités qui donnent lieu à plusieurs descriptions possibles du monde: monde naturel, culturel, existential. On peut envisager dans cette hiérarchisation un premier trait de sa Wiederholung phénoménologique. La condition de possibilité de la capacité de devenir soi-même annoncée en 
98 Claude Romano au carrefour de la phénoménologie française

1998 se transforme en possibilités (au pluriel) en tant que capacités de décrire différemment le monde sans se passer de l'ouverture naturelle et, surtout, sans la rabattre d'emblée sur le monde existential. En effet, avant d'être existential, l'ouverture est ouverture au monde naturel et ensuite ouverture culturelle et, donc, existentiale. La condition de possibilité d'être soi-même, condition irréductible et inaliénable, irrenonçable afin que n'importe quelle chose soit d'abord vécue et ensuite comprise, se fonde sur cette ouverture originaire. Et il s'agit d'une ouverture qui est en-deçà de toute opposition entre théorie et pratique et dont le sens est de s'orienter pratiquement dans le monde. ${ }^{67}$

Une question, cependant, pourrait s'adresser à cette Wiederholung: si la racine, pour ainsi dire, naturelle, de cette capacité de compréhension est repérable, d'où provient-elle celle qui est pratique? Ne faut-il pas chercher encore plus au fond pour trouver ce qui fait que la capacité soit "pratiquement capable"? Encore, cette Wiederholung qui répète ce que de la capacité de l'homme est "irréductible" - et irrenonçable - ne doit-elle pas déceler aussi la capacité de se refuser face à l'irréductible? Si cela n'était pas le cas, ne seraiton pas vis-à-vis d'une sorte d'inéluctabilité pratique naturelle? La question reste ouverte même si on revient aux questions concernant l'anticartésianisme. En effet, notre avis est que, plutôt que vis-à-vis des motifs phénoménologiques classiquestels que l'intentionnalité et l'épochè/réduction, l'anti-cartésianisme le plus radicale se déclare vis-à-vis de l'expérience dont Romano conteste patiemment tout psychologisme phénoménologique jusqu'à aboutir à la proposition d'une expérience nonsubjective qui n'oblige pourtant pas à renoncer à la distinction immanence / transcendance - voire à la distinction conscience-monde:

L'expérience comme ouverture au monde est bien, si l'on veut, subjective, mais elle échappe à toute intimité et à toute intériorité de l'ego. [...] (Il est possible de) rejeter la subjectivité de l'expérience, tout en maintenant la pertinence d'une certaine distinction entre immanence et transcendance au sein du champ - neutre - de l'expérience elle-même. ${ }^{68}$

Romano précise ainsi le sens de l'expérience:

L'expérience en tant que telle, c'est-à-dire en tant qu'ouverture au monde (en moi et hors de moi), n'est pas génétiquement subjective mais relationnelle: elle est la modalité même de ma transcendance incessante avec mon entourage, elle naît pour ainsi dire en ce point de jonction où mon corps se projette sur les choses, elle est l'événement continué de cette rencontre sous les auspices d'un corps agile et agissant." 69

Journal of French and Francophone Philosophy | Revue de la philosophie française et de langue française Vol XXI, No 2 (2013) | http://www.jffp.org | DOI 10.5195/jffp.2013.605 
Voilà pourquoi "l'expérience est neutre à l'égard de la distinction du génétiquement subjectif et objectif; structurée selon deux pôles complémentaires, elle est toujours à la fois expérience de soi et du monde, expérience de soi dans le monde et expérience du monde par référence à soi."70 Or si toute apparition et manifestation phénoménologique n'est possible que par l'ouverture au monde, l'expérience subit une métamorphose qui en décèle sa forme la plus propre, en la transformant de "expérience de quelqu'un" en "expérience que je fais" mais qui "ne m'appartient en rien (car) elle n'est ni moi ni en moi, elle est plutôt la manière même dont le monde s'ouvre pour moi, abrupt vertical, insoupçonné." ${ }^{71}$

Cette Wiederholung de l'expérience cherche à deviner le noyau de celle-ci dans la solidarité "nécessaire à toute description de l'expérience comme telle entre un sujet corporel qui est toujours déjà au monde et un monde qui est toujours déjà ouvert à ce sujet antérieurement à toute ses opérations constituantes et/ou configuratrices de possibles, [...] cette co-appartenance en vertu de laquelle l'être humain et le monde forment les deux fils d'une même trame phénoménale sans qu'aucun puisse être isolé comme une instance préordonnée à l'autre." ${ }^{\prime 72}$ Or notre avis est que cette répétition de l'expérience représente le sens de ce que nous avons appelé l'irréductible sans réduction car seule cette expérience rend possible toute manifestation/apparition possible du monde - et donc elle est irréductible en marquant cette même manifestation/apparition de son sceau d'irrenonçabilité. Rien n'est pour nous si cette radicalisation expérientielle n'ouvre pas tout accès au monde. Rien n'est pour nous faute de cette révolution anticopernicienne ${ }^{73}$ qui partant découle $\mathrm{d}^{\prime}$ une première révolution anti-cartésienne.

Et pourtant, la question que l'on posait plus haut reste encore ouverte, possible et, pour ainsi dire, répétable: où s'enracine toute possibilité de se refuser ainsi que de refuser cet irréductible? Serions-nous arrivés à une sorte de nécessitarisme mondanéisant? Ne risquerait-on pas - faute d'une réflexion sur le caractère irréfusable de l'irréductible - de retomber dans la redoutable alternative opposant monde-de-la-vie/monde existential et monde naturel? Au carrefour de la phénoménologie française nous avons retrouvé le "monde": c'est sa réinterprétation qui emmène Romano le long de son parcours. Il traverse de l'intérieur la phénoménologie et il la répète pour en dégager ses puissances nouvelles. Sans doute, son endurante réflexion sur l'"ouverture au monde" répète-t-elle et saisit-elle quelque chose d'irréductible qui mène Romano jusqu'au dépassement de ce qu'il répète. Or il se peut que ce dépassement ne devrait pas concerner la seule phénoménologie - déjà croisée avec l'herméneutique ${ }^{74}$ - mais encore qu'il devrait concerner en même temps une conception mondanéisante - au moins c'est notre avis - galvaudée. Une pensée radicale de l'irréductible sans réduction devrait se méfier de tout nécessitarisme phénoménologique-

Journal of French and Francophone Philosophy | Revue de la philosophie française et de langue française Vol XXI, No 2 (2013) | http://www.jffp.org | DOI 10.5195/jffp.2013.605 
100 Claude Romano au carrefour de la phénoménologie française

mondanéisant qui risquerait de soumettre à ce même nécessitarisme celui pour lequel n'importe quelle chose advient. Romano côtoie bel et bien le problème, remarquant sans cesse qu'il est toujours question de relation relation que l'intentionnalité est en elle-même, relation homme-monde, etc. Plus que l'ouverture au monde, donc, l'essentiel semblerait être la relation avec ce monde. Et pourtant, pourquoi ne pas franchir un pas au-delà du monde lui-même, afin de penser même la refusabilité de l'irréductible et de renoncer à tout nécessitarisme de la mondanéisation?

En soulignant l'exigence de sauvegarder la refusabilité de l'irréductible, nous ne songeons pas au trait moral ou éthique du refus: au contraire, nous envisageons la possibilité même d'en être capable - c'est-à-dire d'être capable de refuser n'importe quelle chose - ou, ce qui revient au même, nous envisageons ce qui fait la possibilité même d'être tout court capable. En effet, on n'est pas capable parce qu'on est en relation avec le monde et la capacité n'est pas ouverture au monde; on est "capable" parce que l'on est tout court - sans relation ou ouverture préalable, ce qui ne se prouve qu'indirectement par la possibilité de se refuser à cette même relation. La refusabilité de l'irréductible n'a donc rien de moral, il s'agit d'une possibilité radicale de liberté vis-à-vis du monde pour atteindre la racine même de l'ouverture "sans" le monde. Mais ouverture à quoi? Comment penser l'irréductibilité de l'ouverture faute d'un terme sur lequel cette même ouverture percerait?

Renversons la question: non pas ouverture-au-monde mais "au monde par (l')ouverture-en-tant-que-telle." Ce renversement impliquerait que l'ouverture en tant que telle n'est possible que sans son propre "au," voire sans une préposition lui appartenant en propre. Disposons-nous d'une figure qui nous permette de penser une ouverture conçue de telle sorte? Oui, nous disposons de la figure du seuil. Aimé par les poètes, ${ }^{75}$ le seuil annonce la possibilité de faire passer et transiter sans s'arrêter ${ }^{76}$; il est ouverture en tant que telle; il est la pure possibilité de passer et donc l'institution même de l'ouverture irréductible et en même temps refusable (car on peut se refuser de passer). Le seuil serait donc ce qui permettrait de repenser non seulement le monde, mais encore la racine à jamais possible de notre irrenonçable ouverture au monde - ouverture irrenonçable (car il s'agit de ce qui nous caractérise en propre) et pourtant refusable (car on est capable de passer sans y être naturellement obligé). C'est sur ce seuil - cœur irréductible du passage - que nous a menés la Wiederholung que Romano propose de la phénoménologie. Plus radicale que le monde est la capacité de passer dans ce monde; plus radicale que l'ouverture au monde est l'ouverture en tant que telle, à savoir cette ouverture qui fait que la phénoménologie puisse franchir des voies nouvelles.

Dès lors se renoncer à l'irréductible ne serait en rien un refus moral: il s'agirait d'un geste entièrement phénoménologique, censé révéler la racine de notre même capacité d'être au monde. C'est par ce geste que se manifeste

Journal of French and Francophone Philosophy | Revue de la philosophie française et de langue française Vol XXI, No 2 (2013) | http://www.jffp.org | DOI 10.5195/jffp.2013.605 
la brisure intime rendant possible notre ouverture au monde. Et c'est par cette ouverture que nous sommes faits capables de traverser ce seuil qui est le monde. De commencement en commencement, selon des commencements qui n'auront jamais de fin.

1 Dans les articles "Naissance et origine," Transversalités, n. 46 (2005): 1-18) et “Un nuovo inizio è ancora possibile? Fenomenologia, ermeneutica, evento," dans C. Romano, Il possibile e l'evento. Introduzione all'ermeneutica evenemenziale, a cura di Auteur (Milano: Mimesis, 2010, 7-17), nous avons abordé les questions concernant la "phénoménologie herméneutique" que Romano a présenté dans les ouvrages L'événement et le monde (Paris: PUF, 1998), L'événement et le temps (Paris: Puf 1999), le recueil d'article /l y a (Paris: PUF, 2003) et Le chant de la vie. Phénoménologie de Faulkner (Paris: Gallimard, 2005).

${ }^{2}$ Claude Romano, Au cœur de la raison, la phénoménologie (Paris: Gallimard, 2010).

3 Même si, comme le remarque Paul Ricoeur, la phénoménologie "s'est fait reconnaître en France" par le thème de l'intentionnalité husserlienne, on ne peut non plus méconnaître le rôle central que la réduction a revêtu dans la phénoménologie de Levinas, Henry, et surtout Marion. Voir Paul Ricoeur, Réflexion faite. Autobiographie intellectuelle (Paris: éditions Esprit 1995, 17).

${ }^{4}$ Cfr. Edmund Husserl, Husserliana XV (Leuven: Springer), 549-556. Le texte en allemand et la traduction en français ont été publiés dans Dominique Janicaud (éd.), L'intentionnalité en question (Paris: Vrin, 1995), 132-145.

5 Jean-Luc Marion, Réduction et donation. Recherches sur Husserl, Heidegger et la phénoménologie (Paris: PUF, 1989), 302.

${ }^{6}$ Voir Marc Richir, Phénoménologie en esquisse. Nouvelles fondations (Grenoble: Jérôme Million, 2000), 474 ss.

${ }^{7}$ Richir, Phénoménologie en esquisse, 478.

${ }^{8}$ Husserl, Intentionnalité et subjectivité, 161.

9 Marc Richir, Méditations phénoménologiques (Grenoble: Jérôme Million, 1992), 102.

${ }^{10}$ Richir, Phénoménologie en esquisse, 480.

${ }^{11}$ Richir, Phénoménologie en esquisse, 72. 
102 Claude Romano au carrefour de la phénoménologie française

12 Eugen Fink, VI. Cartesianische Meditation. Teil 1. Die Idee einer transzendentalen Methodenlehre (Dordrecht: Kluwer Academic Publishers, 1988),

72.

${ }^{13}$ Fink, VI. Cartesianische Meditation, 69-70.

${ }^{14}$ Fink, VI. Cartesianische Meditation, 73.

${ }^{15}$ A ce propos Husserl écrit: "On se trouve devant l'énigme de savoir comment doit (soll) être possible la connaissance du monde en général, mais aussi simplement, la connaissance de l'être au monde" (Husserl, Intentionnalité et être-au-monde, 138).

${ }^{16}$ Husserl, Intentionnalité et être-au-monde, 140.

${ }^{17}$ Husserl, Intentionnalité et être-au-monde, 140.

${ }^{18}$ Husserl, Intentionnalité et être-au-monde, 142.

${ }^{19}$ Husserl, Intentionnalité et être-au-monde, commentaire de Marc Richir, 161.

20 Romano, Au cœur de la raison, la phénoménologie, 514.

${ }^{21}$ Romano, Au cœur de la raison, la phénoménologie, 516.

22 Romano cite E. Fink, De la phénoménologie, tr. fr. par D. Frank (Paris: éditions de Minuit, 1974), 146.

23 Romano, Au cœur de la raison, la phénoménologie, 522-523.

24 Romano, Au cœur de la raison, la phénoménologie, 523.

${ }^{25}$ Cette critique du cartésianisme en tant que seule voie de la phénoménologie est formulée à maintes reprises dans le volume dont nous nous occupons. A titre d'exemple voire 13 , 484 , etc.

${ }^{26}$ Romano, Au cœur de la raison, la phénoménologie, 531.

27 Romano, Au cœur de la raison, la phénoménologie, 532.

${ }^{28}$ Romano, Au cœur de la raison, la phénoménologie, 533.

29 Romano, Au cœur de la raison, la phénoménologie, 534.

30 Richir, Phénoménologie en esquisse, 479: "On dira que cette feinte originaire, hyperbolique, ne produit que de la fiction - et chez Descartes, c'est la fiction du Malin Génie contre laquelle, cependant, la feinte se reprend dans un cogito assurant la certitude factice d'exister pour celui qui feint."

31 Richir, Phénoménologie en esquisse, 479-480.

32 Romano, L'événement et le monde, 73.

${ }^{33}$ Voir à ce propos Romano, Il y $a$, 55-111.

${ }^{34}$ Voir Janicaud, L'intentionnalité en question.

Journal of French and Francophone Philosophy | Revue de la philosophie française et de langue française Vol XXI, No 2 (2013) | http://www.jffp.org | DOI 10.5195/jffp.2013.605 
35 Voir Edmund Husserl, Ideen zu einen reinen Phänomenologie und phänomenologischen Philosophie. I: Allgemeine Einführung in die reine Phänomenologie (1913) (La Haye: Nijhoff, 1952), § 84.

36 Romano remarque à plusieurs reprises que l'intentionnalité husserlienne se caractérise toujours par cette ambiguïté caractérisant aussi bien les actes de la conscience que l'ouverture de la conscience au monde. Pour cette interprétation, nous renvoyons aux chapitres II, III, XIII, XV de Au cœur de la raison, la phénoménologie.

37 Sur le primat de la perception chez Merleau-Ponty, voir Phénoménologie de la perception (Paris: Gallimard, 1945) aussi bien que Le primat de la perception et ses conséquences philosophiques (Lagrasse: Verdier, 1996).

38 Romano, Au cœur de la raison, la phénoménologie, 538-539.

39 Ce qui est explicitement affirmé dans le ch. II, L'intentionnalité husserlienne ainsi que dans les autres chapitres consacrés à ce motif.

${ }^{40}$ Romano, Le chant de la vie. Phénoménologie de Faulkner.

${ }^{41}$ Renaud Barbaras, Vie et intentionnalité. Recherches phénoménologiques (Paris: Vrin, 2003).

42 Barbaras, Vie et intentionnalité, 10.

43 Barbaras, Vie et intentionnalité, 15.

44 Barbaras, Vie et intentionnalité, 18.

45 Barbaras, Vie et intentionnalité, 19.

${ }^{46}$ Barbaras, Vie et intentionnalité, 20.

47 Barbaras, Vie et intentionnalité, 21.

${ }^{48}$ Barbaras, Vie et intentionnalité, 21 (pour les deux citations).

${ }^{49}$ Barbaras, Vie et intentionnalité, 21.

${ }^{50}$ Barbaras, Vie et intentionnalité, 22-23.

${ }^{51}$ Barbaras, Vie et intentionnalité, 23 (pour les deux citations).

52 Romano, Au cœur de la raison, la phénoménologie, ch. XVII, L'horizon (le holisme de l'expérience I), ch. XVIII Le monde (le holisme de l'expérience II), 642726.

53 Romano, Au cœur de la raison, la phénoménologie, 583, nous soulignons.

54 Romano, Au cœur de la raison, la phénoménologie, 585.

55 Romano, Au cœur de la raison, la phénoménologie, 600.

${ }^{56}$ Barbaras, Vie et intentionnalité, 14.

${ }^{57}$ Faute d'une conception adéquate de l'irréductible, on ne pourrait pas sortir des alternatives telles que, par exemple, celle qui oppose entre elles la définition de 
104 Claude Romano au carrefour de la phénoménologie française

l'ego découlant de l'affirmation hobbesienne "homo homini lupus" et celle de l'ego amans, etc.

58 Douglas Richard Hofstadter, Gödel, Escher, Bach: les Brins d'une Guirlande Eternelle, tr. J. Henry et R. French (Paris: InterEditions, 1985), 12.

59 La troisième partie de L'événement et le monde est entièrement consacrée à l'expérience et à son sens phénoménologique.

${ }^{60}$ Cette répétition était le but non seulement de Heidegger mais aussi de Marion que, dans Réduction et donation écrivait: "On ne dépasse pas un véritable pensée en la réfutant, mais en la répétant, voire en lui empruntant les moyens de penser avec elle au-delà d'elle. Alors même l'échec réussit" (10).

${ }^{61}$ C'est le but de Richir.

${ }^{62}$ Romano, L'événement et le monde, 214-215.

${ }^{63}$ Romano, L'événement et le monde, 215.

${ }^{64}$ Romano, L'événement et le monde, 215.

${ }^{65}$ Romano, Au cœur de la raison, la phénoménologie, 719.

${ }^{66}$ Romano, Au cœur de la raison, la phénoménologie, 719-720.

${ }^{67}$ Romano, Au cœur de la raison, la phénoménologie, 614-615.

68 Romano, Au cœur de la raison, la phénoménologie, 804.

${ }^{69}$ Romano, Au cœur de la raison, la phénoménologie, 809.

${ }^{70}$ Romano, Au cœur de la raison, la phénoménologie, 809.

71 Romano, Au cœur de la raison, la phénoménologie, 823.

72 Romano, Au cœur de la raison, la phénoménologie, 944.

73 Voire Romano, Au coeur de la raison, la phénoménologie, ch. XXIII, Une révolution anti-copernicienne: le monde de la vie, 907 ss.

74 A titre d'exemple voir, sur ce sujet, Claude Romano, "L'horizon de la phénoménologie," Iris. Annales de philosophie 28 (2008): 1-37, notamment 37.

${ }^{75}$ Voir par exemple Yves Bonnefoy, Dans le leurre du seuil, ou la IX élégie de Duino de Rainer Maria Rilke.

${ }^{76}$ Une tentative philosophique de réfléchir sur le passage - plus que sur le seuil - est le collectif Le souci du passage. Mélanges offertes à Jean Greisch (Paris: Cerf, 2004).

Journal of French and Francophone Philosophy | Revue de la philosophie française et de langue française Vol XXI, No 2 (2013) | http://www.jffp.org | DOI 10.5195/jffp.2013.605 